\title{
Rede Óptica Comutada por Circuito e por Rajada
}

\author{
Eduardo M. G. de Queiroz, Helvécio M. Almeida e Amílcar C. César
}

\begin{abstract}
Resumo - Apresentamos neste artigo um algoritmo de roteamento e alocação de comprimento de onda de tráfego em arquitetura híbrida de rede óptica (OHS - optical hybrid switching) comutada por circuito (optical circuit switching) e por rajada (optical burst switching). O tráfego das duas redes é roteado separadamente em dois conjuntos de comprimentos de onda. Esta abordagem heurística permite melhorar o desempenho da rede OHS em relação à técnica na qual os dois tipos de tráfego disputam indistintamente o mesmo recurso. Na rede OCS o tráfego é distribuído em seis classes de taxa de bit. A geração de tráfego nas duas redes é equalizada. As simulações computacionais sobre a configuração da rede NSFNet mostram a viabilidade da abordagem.
\end{abstract}

Palavras-chave — rede híbrida, rede comutada por circuito, rede comutada por rajada, roteamento, alocação de largura de faixa.

\begin{abstract}
In this article we proposed an algorithm for traffic routing and wavelength assignment in an optical hybrid switching (OHS) combining optical circuit switching (OCS) and optical burst switching (OBS). The traffic of the two networks is routed separately in the two sets of wavelengths. This improves network performance in relation to the OHS technique in which the two types of traffic dispute the same resource. The OCS network traffic is distributed in six classes of bit rate. The generation of the traffic on both networks is equalized. The computational simulations on the NSFNet network topology show the viability of the heuristic approach.
\end{abstract}

Keywords - optical hybrid switching (OHS), optical burst switching (OBS), optical circuit switching (OCS), traffic routing, bandwidth assignment.

\section{INTRODUÇÃO}

O sucesso da Internet produziu aumento expressivo do tráfego nas redes de telecomunicações e transformou o IP (Internet protocol) em padrão de aplicações e arquitetura de rede.

$\mathrm{Na}$ camada eletrônica os roteadores IP processam pacotes, que são blocos de bits de cabeçalho (endereçamento e correção de erros) e payload (dados de usuários). O tráfego entre nós origem e destino são fracionados em pacotes que

Eduardo M. G. de Queiroz, Helvécio M. Almeida e Amílcar C. César, Universidade de São Paulo, Escola de Engenharia de São Carlos, Depto. Eng. Elétrica, São Carlos, SP. e-mails: emartinelli@usp.br; asah@terra.com.br; amilcar@sc.usp.br. Esta pesquisa foi parcialmente financiada pela Capes e CNPq (processo 474584/2007-0) são roteados compartilhando recursos da rede e evitando desperdício. A "granularidade", o menor elemento de informação manipulável, é o pacote.

Por outro lado, a camada física se consolidou pelo uso da fibra óptica e da técnica WDM (wavelength division multiplexing), que é a justaposição de vários comprimentos de onda. Os comutadores ópticos (optical crossconnectOXC) desempenham função de comutar todos os comprimentos de onda de uma fibra para outra, grupos de comprimentos de onda (waveband) ou comprimento de onda individual.

A conexão entre nós origem e destino (ligthpath) é estabelecida por meio de comprimentos de onda. A técnica WDM e a natureza dos dispositivos ópticos associados permitem processar apenas comprimento de onda. Como a "granularidade" deste sistema é o comprimento de onda, a comutação na camada óptica é semelhante à comutação por circuito das redes eletrônicas clássicas de comunicação, nas quais um conjunto de recursos é reservado para o estabelecimento de conexão. Os vários comprimentos de onda são utilizados para conectar diferentes pares de nós.

$\mathrm{Na}$ rede roteada por comprimento de onda uma demanda de tráfego, mesmo exigindo largura de faixa menor que a máxima, ocupa um comprimento de onda. Neste caso, a "sobra" de banda indica ocupação ineficiente.

Uma forma de evitar desperdício de banda é agregar (grooming) tráfego na camada eletrônica de tal forma que somente tráfego "banda cheia" ocupe comprimentos de onda. Este processo exige conversão óptica-eletrônica-óptica (OEO).

A demanda por largura de faixa e a necessidade de otimizar o uso dos recursos das redes deu origem ao interesse de compatibilizar a flexibilidade dos pacotes e a transparência da rede puramente óptica, por meio da comutação de pacotes diretamente no domínio óptico.

$\mathrm{Na}$ camada óptica há três técnicas básicas de comutação: por circuito (OCS - optical-circuit switching), por rajadas (OBS — optical burst switching) e por pacotes (OPS — optical packet switching) [1].

A técnica OCS é baseada em comprimentos de onda. A técnica OBS é baseada na agregação dos pacotes IP dos usuários realizada pelos roteadores de ingresso situados na borda da rede, formando rajadas. O cabeçalho é processado eletronicamente. Em rede OPS, pacotes IP formam o payload de um pacote óptico que possui um cabeçalho óptico. No nó de comutação o cabeçalho é interrogado e suas informações são utilizadas para configurar o trecho seguinte de 
roteamento. Embora a técnica OPS seja a solução mais compatível com a IP e ofereça a menor "granularidade" e utilização de largura de banda, ela exige dispositivos ópticos para realizar chaveamento da ordem de nanossegundos e buffer para reduzir o bloqueio de pacotes em caso de congestionamento.

O cenário futuro mais provável é aquele no qual a infraestrutura óptica será compartilhada por usuários de serviços residenciais, de negócios e científicos, exigindo bandas diferentes e operando vários tipos de protocolos. Para tanto, uma solução atraente é a combinação de redes OCS e OBS [2]-[3], que prenuncia ser o horizonte mais próximo, enquanto OPS seria o mais distante, por causa de limitação de características de dispositivos.

Se aceitarmos que a arquitetura de rede óptica do futuro é a comutada por pacotes, então a sequência de progressão tecnológica é passar da rede OCS atual para rede OBS e, finalmente, para a OPS. A rede híbrida pode ser entendida como estágio intermediário de progressão entre redes puras OCS e OBS.

A natureza dos tráfegos OCS e OBS é diferente. Os dispositivos que estão alocados nos nós da rede são especializados e processam rajadas e conexões separadamente. Assim, uma forma de rotear os dois tráfegos em redes híbridas é separar os tráfegos em comprimentos de onda diferentes.

Com objetivo de agregar as vantagens de cada tipo de rede, as arquiteturas híbridas (optical hybrid switching - OHS), aquelas capazes de processar tráfego OCS e OBS na mesma arquitetura de nó, são as mais promissoras [1]-[6].

Neste artigo, descrevemos um algoritmo que fornece solução heurística para o problema de alocação de recursos em rede OHS. Os nós desta rede são capazes de comutar comprimentos de onda e rajadas separadamente em dois conjuntos de comprimentos de onda. Os nós são capazes de realizar agregação de tráfego na camada eletrônica para ocupar eficientemente a banda dos comprimentos de onda da rede híbrida.

A contribuição principal desta abordagem de rede híbrida é rotear tráfegos OBS e OCS em comprimentos de onda exclusivos para melhorar o desempenho global da rede. Nas abordagens convencionais os comprimentos de onda são utilizados alternada e indistintamente para os dois tipos de tráfegos. Analisamos também o desempenho de uma rede OCS e uma rede puramente OBS, que aloca caminhos ópticos para rotear pacotes de acordo com tabela de roteamento dos nós intermediários. Neste caso, nossa proposta estende os resultados obtidos em [4], que utiliza apenas uma taxa de bit para a rede OCS. Utilizamos seis taxas de bit, de acordo com os padrões SONET/SDH.

Este artigo está organizado da seguinte maneira. Na Seção II é realizada uma descrição das redes OCS, OBS, da rede híbrida, da arquitetura dos nós e da modelagem de tráfego. $\mathrm{Na}$ Seção III é apresentada a abordagem. Na Seção IV é realizada uma comparação entre redes OCS e puramente OBS e são apresentados os resultados numéricos obtidos por meio de simulação computacional. Finalmente, na Seção V são apresentadas as conclusões do trabalho.

\section{REDE HÍBRIDA}

A rede híbrida investigada está mostrada na Figura 1. As características dos diferentes tipos de rede estão descritas a seguir.

\section{1) Rede OCS}

A técnica OCS executa o roteamento de tráfego entre os nós origem e destino por meio dos comprimentos de onda. As rotas (ligthpaths) são formadas pelos vários enlaces interconectando os nós da rede. Os nós são compostos por OXCs, multiplexadores e demultiplexadores WDM, dispositivos add-drop e transceptores ópticos. Com estes dispositivos o nó está apto a realizar agregação de tráfego na camada eletrônica.

O roteamento e alocação do comprimento de onda são realizados em duas etapas. Na primeira, o menor caminho óptico entre os nós origem e destino é determinado por meio do algoritmo Dijkstra. Na segunda etapa, um comprimento de onda disponível é alocado para o tráfego por meio do algoritmo first-fit.

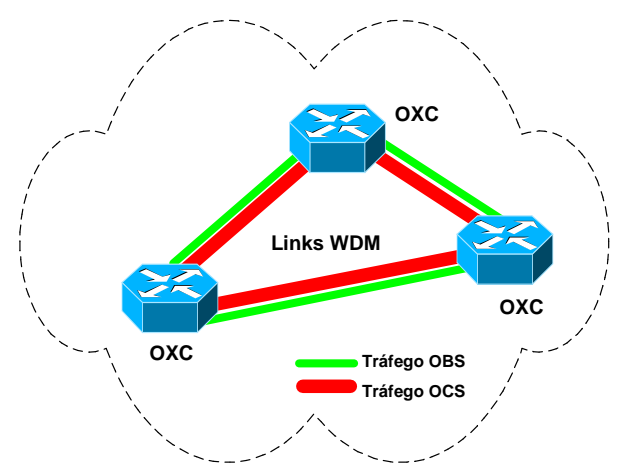

Figura 1. Arquitetura da rede híbrida analisada neste artigo. OXC: Optical cross-connect; WDM: wavelength division multiplexing; OCS: Optical circuit-switching; OBS: Optical burst-switching.

\section{2) Rede $O B S$}

A técnica OBS é baseada na formação de rajadas por meio da agregação de pacotes IP dos usuários realizada pelos roteadores de ingresso situados na borda da rede. A agregação é realizada com base em parâmetros como destino (critério mais comum) ou classe de serviço. Cada rajada é associada a um pacote de controle (cabeçalho) que transporta informações como comprimento da rajada e roteamento. O cabeçalho é processado eletronicamente nos nós intermediários para reserva de recurso, enquanto a rajada é roteada no domínio óptico. Em alguns nós da rede pode haver competição entre rajadas por recursos, como mesma fibra e/ou comprimento de onda. Neste caso, esquemas para resolver o problema de contenção devem ser empregados [4]. Os algoritmos de agregação de pacotes se baseiam em tempo ou limiar, ou em ambos. A partir da agregação de pacotes, uma rajada é criada e esta é a "granularidade" da rede OBS que, portanto, opera em subcomprimento de onda, diferentemente da rede OCS totalmente óptica. 
Sendo assim, a premissa da rede OBS é habilitar e desabilitar dinamicamente rotas para o tráfego da rajada, do mesmo modo que para o caso de comutação por circuito, mas por período curto, necessário para a transmissão da rajada [5]. As rajadas que trafegarão pela rede OBS são oriundas de rede de grandes flutuações de tráfego, como rede metropolitana.

No caso de uma rede puramente OBS, como a que será apresentada na Seção IV, o roteamento é realizado por meio da procura de comprimento de onda com largura de faixa suficiente para alocar rajadas, com base na tabela de roteamento do nó. Esta tabela contém, para cada nó, os comprimentos de onda e suas respectivas bandas disponíveis aos nós adjacentes conectados. Este procedimento é interrompido quando o nó destino da rajada é alcançado ou o algoritmo executa procedimento de busca por um período de tempo muito longo. Nestes casos, a rajada é descartada.

\section{3) Rede híbrida OCS e OBS (OHS)}

A rede híbrida agrega a técnica OCS e OBS na mesma arquitetura, como mostra a Figura 1. Como a natureza dos tráfegos OCS e OBS é diferente, em nossa abordagem eles são roteados em comprimentos de onda diferentes.

\section{4) Arquitetura dos Nós}

Utilizamos em nossa simulação a topologia da rede NSFNet, que é mostrada na Figura 2.

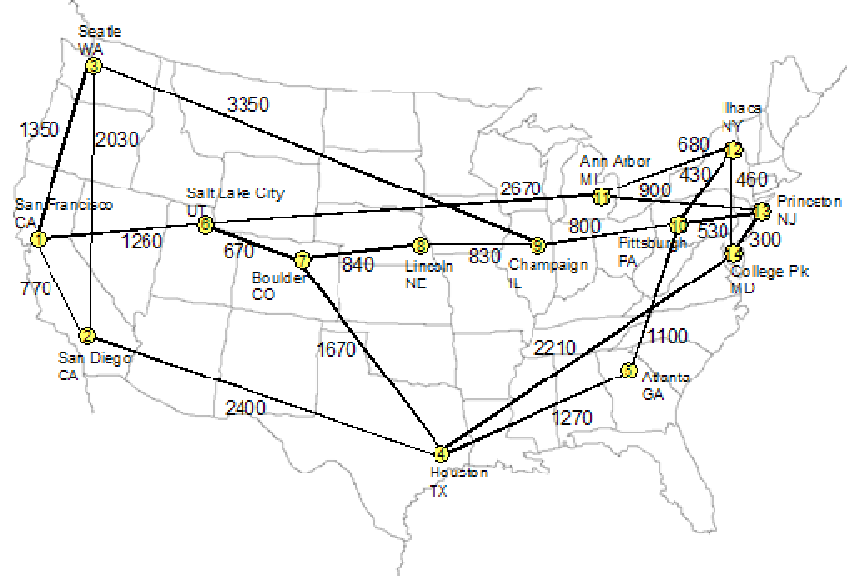

Figura 2. Rede NSFNet.

Em cada enlace há 8 comprimentos de onda disponíveis com capacidade máxima 10 Gbps. Dos 14 nós, 9 são capazes de realizar agregação de tráfego com fator 64 [7]. Os nós são $1,3,5,6,7,9,11,13$ e 14 . Assim, no caso de tráfego OCS, 64 conexões de 155,52 Mbps ou 16 conexões de 622,08 Mbps são alocadas na capacidade máxima de um comprimento de onda, $10 \mathrm{Gbps}$. O tráfego gerado em um destes nós é agregado na camada eletrônica e ocupa o primeiro comprimento de onda que disponha de banda. Os outros 5 nós $(2,4,8,10$ e 12) não realizam agregação de tráfego. O tráfego gerado neste nó é alocado em um comprimento de onda livre qualquer que seja sua taxa de bit. $\mathrm{O}$ tráfego que não se destina a este tipo de nó é comutado pelo OXC para o nó destino.

\section{5) Modelagem de Tráfego}

O tráfego da rede híbrida OCS-OBS simulada é composto por conexões OCS e rajadas OBS. A análise de desempenho das duas redes somente pode ser feita adequadamente se houver normalização da geração de tráfego. A normalização adotada em nossa simulação segue a relação $\rho=\lambda /(W \mu)$, na qual $\lambda$ é a quantidade de conexões por segundo, $W$ é o número de comprimentos de onda e $\mu$ é a média de duração das conexões [4].

As solicitações de conexão OCS seguem distribuição de Poisson e têm probabilidade de ocorrência $p_{c}=70 \%$ e o intervalo médio de duração é $\mu=60 \mathrm{~s}$. $\mathrm{O}$ tráfego gerado nos nós é distribuído em 6 tipos de acordo com a taxa de bit, conforme mostra a Tabela I. Solicitações de conexão com taxas mais baixas são mais prováveis de ocorrer.

\begin{tabular}{ccc}
\multicolumn{2}{c}{ TABELA I. DISTRIBUIÇÃO DAS LARGURAS DE FAIXA POR TRÁFEGO (OCS) } \\
\hline $\begin{array}{c}\text { Tipo de } \\
\text { Tráfego }\end{array}$ & Taxa de Bit (Mbps) & Probabilidade de Ocorrência (\%) \\
\hline I & 155,52 & 59 \\
II & 622,08 & 15 \\
III & 933,12 & 10 \\
IV & 1.244 & 8 \\
V & 2.488 & 5 \\
VI & 10.000 & 3 \\
\hline
\end{tabular}

Para a rede OBS, adotamos modelo de tráfego autossimilar com parâmetro Hurst $H=1,35$ e número mínimo de pacotes 10.000 e máximo 600.000. O tamanho mínimo de pacote é 64 bytes e máximo 1.500 bytes.

No caso da rede puramente OBS, cujos resultados das simulações são apresentados na subseção IV.A, o roteamento é baseado na tabela de roteamento de cada nó intermediário.

No caso da rede OBS, cujos resultados das simulações são apresentados na subseção IV.B (rede híbrida), a probabilidade de ocorrência é $p_{b}=30 \%$ e o intervalo médio de duração é $\mu=80$ s. O menor caminho também é determinado por meio do algoritmo Dijkstra. Estes parâmetros e procedimento são os adotados em [3].

Para cada solicitação de conexão OCS ou rajada OBS na rede híbrida, o algoritmo procura por um comprimento de onda que possa alocar a largura de faixa especificada no caminho óptico [3].

No caso de conexão OCS, a largura de faixa do tráfego gerado é a mostrada na Tabela I. No caso de rajada OBS, a largura de faixa é variável e depende da geração do tráfego autossimilar.

\section{AlOCAÇÃo DE COMPRIMENTO DE ONDA EM REDES HÍBRIDAS}

Neste trabalho propomos uma técnica de roteamento para rede híbrida OCS-OBS que separa os dois tipos de tráfego em comprimentos de onda distintos. Depois de várias simulações com diferentes configurações de reserva de comprimentos de onda para tráfego OBS e OCS, escolhemos alocar as conexões OCS em 5 comprimentos de onda, 1, 2, 3, 4 e 5, e as rajadas OBS em 3 comprimentos de onda, 6, 7 e 8. Esta foi a configuração que apresentou o melhor desempenho depois 
de serem realizadas inúmeras simulações. Para a escolha dos comprimentos de onda nas faixas especificadas é utilizado o algoritmo first-fit. O objetivo deste procedimento é organizar a ocupação da largura de faixa dos comprimentos de onda e, conseqüentemente, separar os comportamentos distintos de tráfego. A Figura 3 ilustra o procedimento.

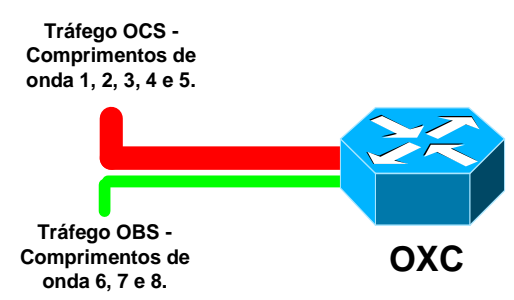

Figura 3. Técnica de separação de tráfego OCS e OBS.

\section{RESULTADOS NUMÉRICOS}

Nesta seção são apresentados os resultados numéricos obtidos por meio de simulação computacional. O programa foi escrito em linguagem Matlab e processado em computador Core 2 Quad Q660, com 4GB. Cada ponto dos gráficos foi obtido em cerca de 20 minutos.

\section{A. Desempenho de Redes Puramente OBS e OCS}

As redes OBS e OCS são analisadas separadamente, adotamos 8 comprimentos de onda disponíveis em cada rede e o tráfego gerado nos nós da rede está especificado na Subseção II.5.

A probabilidade de bloqueio global das redes OBS e OCS é mostrada na Figura 4. A probabilidade de bloqueio da rede OCS é maior que a da rede OBS, com exceção da faixa de carga muito elevada para qual o desempenho das duas converge. Para cargas elevadas, a probabilidade de bloqueio é alta nas duas redes porque a banda disponível nos comprimentos de onda vai se esgotando.

Para cargas pequenas, a rede OCS apresenta probabilidade de bloqueio maior que a OBS porque há 6 tipos de tráfego (ver Tabela I) na rede OCS que competem por banda. As taxas menores, como 155,52 Mbps, são menos bloqueadas, embora ocorram em quantidade maior, porque a capacidade máxima, $10 \mathrm{Gbps}$, acomoda 64 conexões. No entanto, nesta mesma capacidade são alocadas 16 conexões de 622,08 Mbps. Alternativamente, podem ser alocadas 15 conexões de 622,08 Mbps e 4 conexões de 155,52 Mbps. Assim, o algoritmo first-fit vai alocando as solicitações, "preenchendo" eficientemente a banda disponível em cada comprimento de onda. À medida que aumenta a taxa de bit das solicitações, a probabilidade de bloqueio também aumenta. Quatro solicitações de 2.488 Mbps ocupam toda a capacidade. Portanto, as solicitações de maior taxa são relativamente mais bloqueadas, contribuindo para o aumento da probabilidade global.

Por outro lado, na rede OBS as rajadas não competem significativamente quando a carga é baixa. Relativamente, há disponibilidade de recurso com cargas baixas, pois a freqüência de geração de rajadas não é grande e, assim, grupos de rajadas de 2 de 100, uma de 120, 2 de 200, uma de 250, 3 de 300 e 4 de 350 Mbps podem ser alocados em um comprimento de onda e ainda assim haverá banda disponível de 6,730 Gbps. Para cargas elevadas, os tamanhos das rajadas variam da mesma maneira que para cargas baixas, mas são geradas com maior freqüência, requisitando mais banda, que vai se esgotando.

Embora a geração de tráfego para as duas redes tenha sido equalizada (ver subseção II.5), a natureza distinta dos tráfegos dificulta comparação perfeita nos mesmos moldes, já que a rede OBS é caracterizada por grandes flutuações de tráfego.

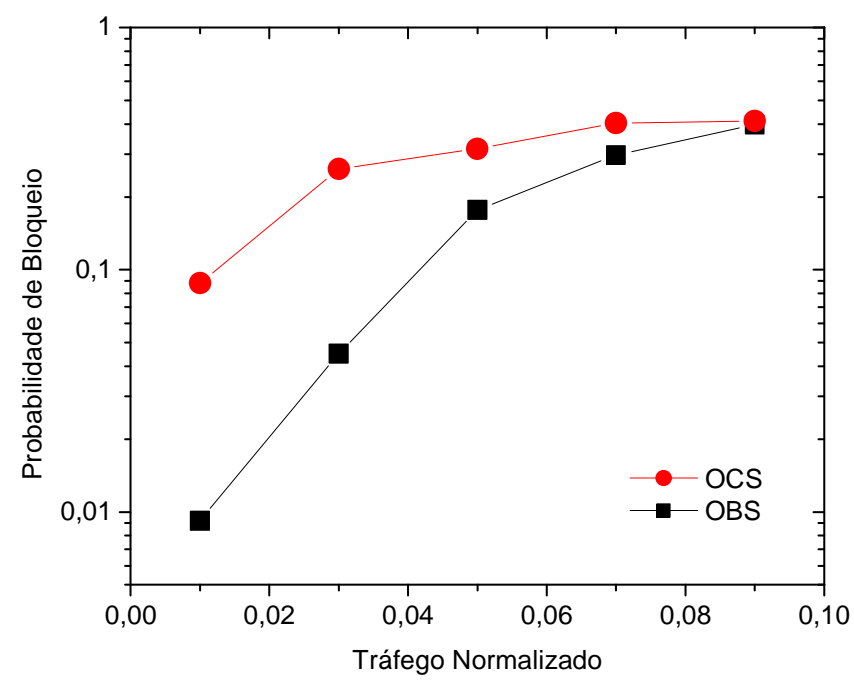

Figura 4. Probabilidade de bloqueio das redes OBS e OCS.

A Figura 5 exibe o throughput (vazão) de tráfego nas duas redes. Apesar de apresentar vazão inferior para tráfegos menores, a rede OCS apresenta um melhor aproveitamento de banda para tráfegos maiores. Este resultado é consequiência da distribuição de larguras de faixa que apresenta tráfego (ver Tabela I) a que a rede OCS é submetida. A ocupação de banda dos comprimentos de onda é mais organizada do que a da rede OBS, caracterizada por grandes flutuações de tráfego.

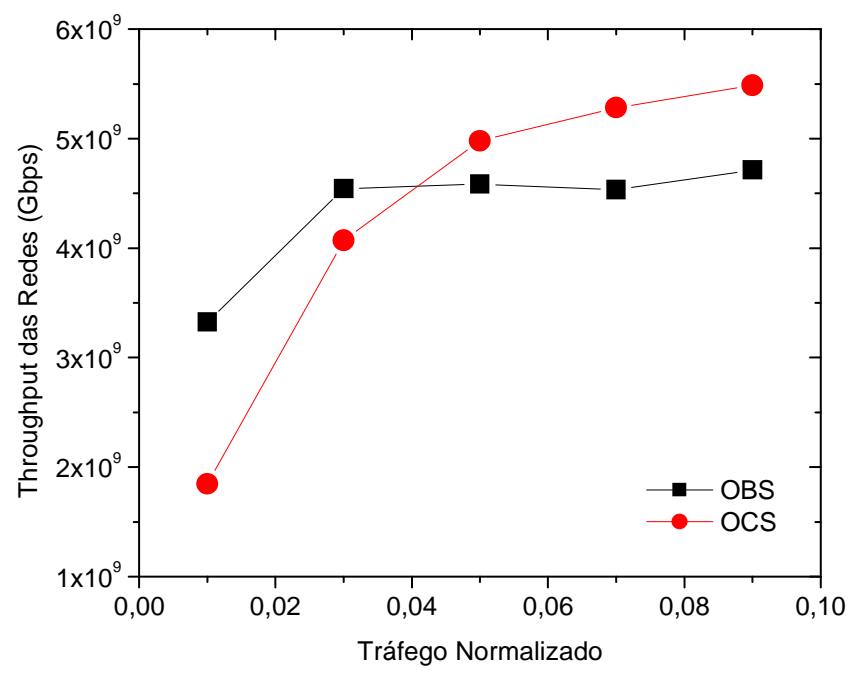

Figura 5. Throughput das redes OBS e OCS. 
Na rede OBS são geradas rajadas que ocupam largura de faixa de, por exemplo, 100 Mbps em um comprimento de onda durante sua duração. No caso da existência de pequena banda livre, sua ocupação é mais difícil porque seria ocupada por rajada pequena, gerada com frequência menor.

Na rede OCS há seis tipos de tráfego com taxa de bit fixa e o algoritmo conta mais possibilidades de acomodação. Se a banda de $10 \mathrm{Gbps}$ for ocupada por 25 conexões de $155 \mathrm{Mbps}$, duas de 2,5 Gbps e uma de 933 Mbps haverá disponibilidade de $192 \mathrm{Mbps}$, que poderá ser ocupada por uma conexão de $155 \mathrm{Mbps}$, que tem $59 \%$ de probabilidade de ocorrência.

No caso do padrão de ocupação de banda na rede OBS, se algum comprimento de onda dispuser de banda de $10 \mathrm{Mbps}$, uma rajada precisa ser menor ou igual a este valor para ser alocada. Porém, a característica da rede simulada é a geração de grandes rajadas, o que dificulta a ocupação daquela banda disponível.

\section{B. Rede Híbrida OCS-OBS}

Nesta seção são apresentados os resultados obtidos para a rede híbrida. Foram simuladas quatro configurações, conforme mostra a Tabela II. No caso da configuração 2, a rede OBS utiliza o mesmo roteamento da rede OCS.

TABELA II. Configurações DE REDE SimULADAS

\begin{tabular}{|c|c|}
\hline Configuração & Descrição \\
\hline (1) & Rede puramente OCS - utiliza Dijkstra para roteamento. \\
\hline (2) & Rede OBS que utiliza algoritmo Dijkstra para roteamento \\
\hline (3) & $\begin{array}{l}\text { Rede híbrida (OHS) OBS-OCS sem o esquema de reserva } \\
\text { de comprimentos de onda - utiliza Dijkstra para } \\
\text { roteamento para os dois tipos de tráfego. }\end{array}$ \\
\hline (4) & $\begin{array}{l}\text { Rede híbrida (OHS) OBS-OCS com o esquema proposto } \\
\text { neste trabalho de reserva de comprimentos de onda - } \\
\text { utiliza Dijkstra para roteamento para os dois tipos de } \\
\text { tráfego. }\end{array}$ \\
\hline
\end{tabular}

A Figura 6 exibe a probabilidade de bloqueio de rajadas OBS na rede híbrida para as configurações (3) e (4). A rede com reserva de comprimentos de onda para tráfego OBS e OCS apresenta desempenho melhor para toda faixa de tráfego normalizado. Se uma rajada de $100 \mathrm{Mbps}$ for gerada na rede paralelamente a uma conexão de $155 \mathrm{Mbps}$, a rajada é alocada em algum comprimento de onda 6 a 8 , enquanto a conexão é alocada em algum comprimento de onda 1 a 5. Desta maneira, como há separação dos tráfegos, não há competição por largura de faixa entre rajadas OBS e conexões OCS no mesmo comprimento de onda. Assim, há separação entre os comportamentos de ocupação de banda dos comprimentos de onda. A reserva favorece a "organização" entre tráfegos de mesma natureza.

A Figura 7 apresenta os resultados da probabilidade de bloqueio em função do tráfego para as quatro configurações descritas na Tabela II. Os resultados para as configurações 3 e 4 da rede OHS incluem rajadas OBS e conexões OCS, diferentemente da Figura 6, na qual os resultados mostram apenas a probabilidade de bloqueio das rajadas OBS da rede híbrida OHS. A configuração 4 proposta (com reserva de comprimento de onda) exibe desempenho melhor para tráfegos pequenos. $\mathrm{O}$ aumento da probabilidade de bloqueio global a partir da carga normalizada 0,004 aumenta porque o número de comprimentos de onda reservados para as conexões OCS da rede OHS é menor do que o da rede OCS com configuração 1 .

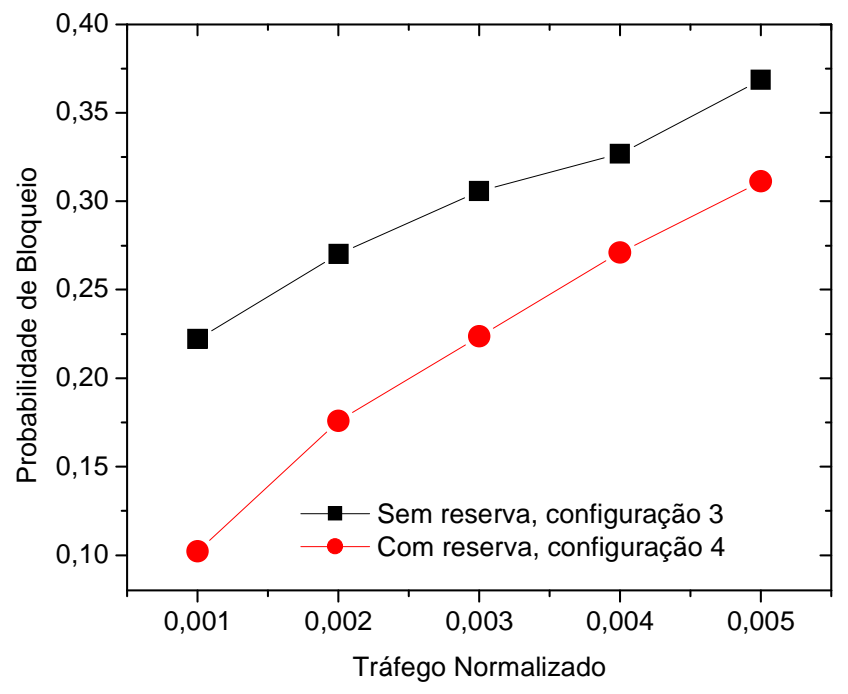

Figura 6. Probabilidade de bloqueio das rajadas OBS em rede híbrida.

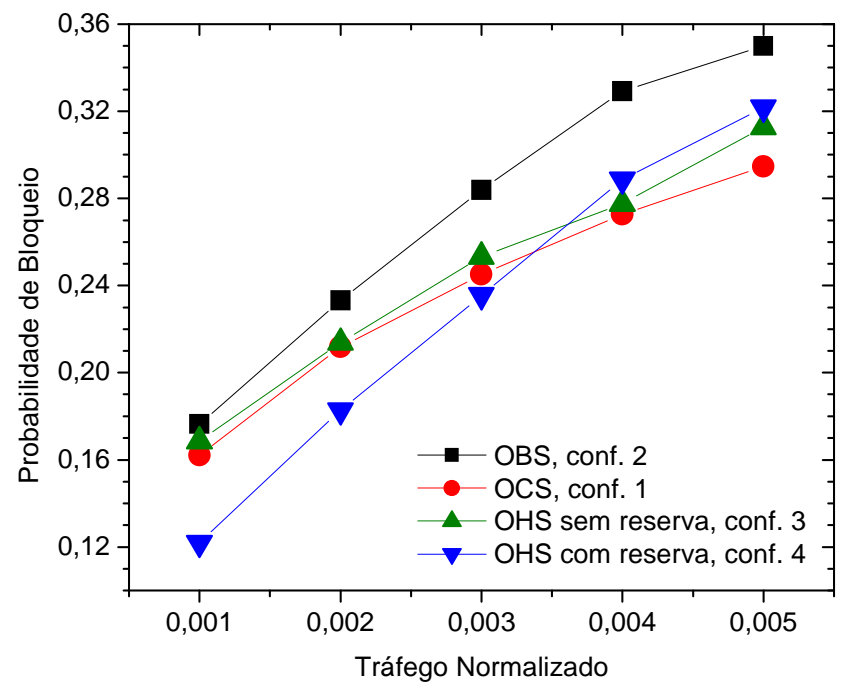

Figura 7. Probabilidade de bloqueio global da rede. As configurações 3 e 4 incluem rajadas OBS e conexões OCS.

Na Figura 8 é exibida a vazão dos comprimentos de onda para as quatro configurações distintas. Observa-se que as duas configurações de rede OHS têm valores maiores do que os da rede OBS com configuração 2 e menores do que os da rede OCS (configuração 1). Desta maneira, o desempenho das configurações da rede OHS situa-se em estágio intermediário em relação ao desempenho da rede OCS (configuração 1) e rede OBS com configuração 2. Este comportamento é consequiência da presença do tráfego de rajadas na rede híbrida, pois a rede OBS apresenta padrão diferente de ocupação de banda e, assim, sua vazão menor de tráfego influencia na diminuição global do throughput da rede OHS. 
Com este tipo de esquema, o objetivo é melhorar a distribuição dos tipos de tráfego da rede e, conseqüentemente, não penalizar as rajadas OBS que utilizam a arquitetura de rede OCS para roteamento.

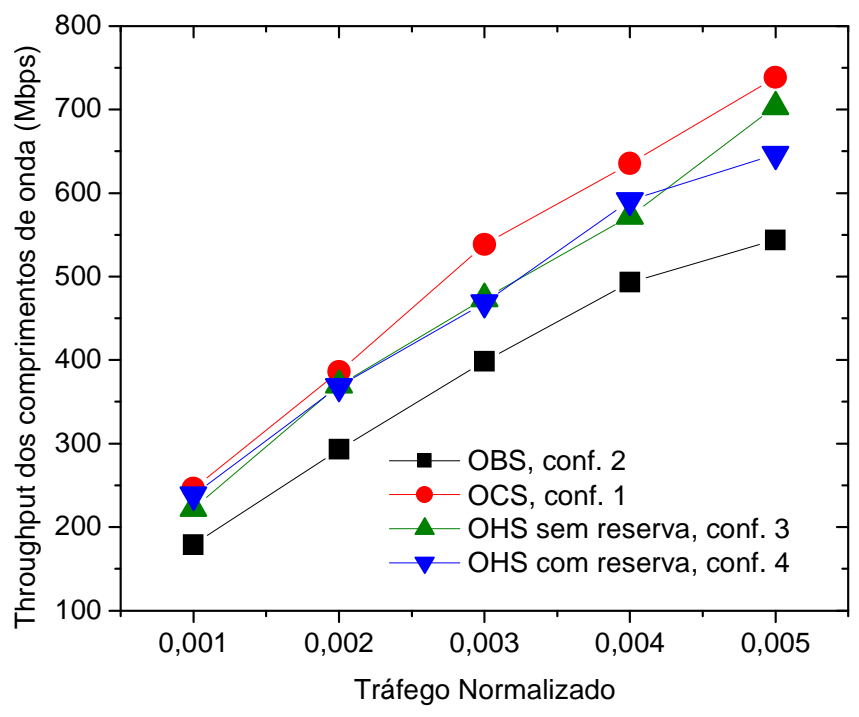

Figura 8. Throughput das redes.

A Figura 9 exibe as probabilidades de bloqueio das seis taxas de bits de tráfego OCS da rede híbrida com reserva (configuração 4) para tráfego normalizado 0,003. A maior probabilidade de bloqueio ocorre para a taxa de $10 \mathrm{Gbps}$, que demanda toda largura de faixa de comprimento de onda. Para os demais tráfegos, as diferenças são pequenas, pois a largura de faixa de 10 Gbps pode suportar várias conexões dos vários tipos de tráfego simulados.

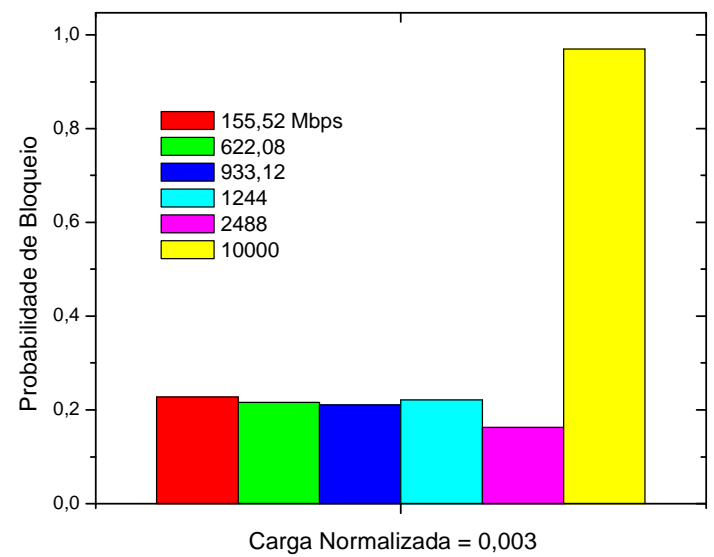

Figura 9. Probabilidade de bloqueio das taxas de bits.

Comparando a rede OCS com a rede puramente OBS, notamos que a rede híbrida apresenta desempenho inferior em termos de probabilidade de bloqueio devido ao menor número de comprimentos de onda específicos para cada tipo de tráfego (OBS e OCS), provocando um maior número de bloqueios de rajadas e conexões. Para as redes OCS e OBS utilizamos 8 comprimentos de onda cada. Assim, a comparação é entre desempenho das duas redes com capacidade máxima de recursos. Para a rede híbrida, são utilizados 5 comprimentos de onda para tráfego OCS e 3 para tráfego OBS. Entretanto, o número de comprimentos de onda pode ser alterado de acordo com taxa de bloqueio desejada.

\section{CONCLUSÕES}

Neste trabalho foram investigadas redes OHS (optical hybrid switching), comportando chaveamento de comprimento de onda (OCS-optical circuit switching) e chaveamento de rajadas de pacotes (OBS-optical burst switching). $\mathrm{O}$ algoritmo proposto roteia separadamente os dois tipos de tráfego em grupos reservados de comprimentos de onda.

O objetivo da rede híbrida é a combinação das vantagens dos dois tipos de tráfego numa mesma arquitetura de nó, proporcionando uma migração tecnológica entre as redes ópticas OCS e OPS. A técnica proposta de separação de tráfego por faixas de comprimento de onda visa atender ao tráfego OBS na arquitetura de rede OCS, diminuindo sua probabilidade de bloqueio. Os resultados mostram que a separação dos tráfegos reduz a probabilidade de bloqueio das rajadas OBS, pois não há competição por largura de faixa entre rajadas e conexões OCS. Para a probabilidade de bloqueio global, a configuração de rede híbrida apresenta os menores valores até certo valor de tráfego normalizado, a partir do qual o menor número de comprimentos de onda reservados para as conexões OCS da rede OHS em relação à rede OCS com configuração 1 contribui para o aumento do bloqueio.

Também foi realizada análise de desempenho das redes puramente OBS e OCS com tráfego exigindo larguras de faixa distintas. Observamos que o padrão de ocupação dos comprimentos de onda proporcionado pelo tráfego da rede OCS resulta em throughput maior do que na rede OBS, para cargas elevadas.

\section{REFERÊNCIAS}

[1] A. Agrawal, T. S. El-Bawab e L. B. Sofman, "Comparative Account of Bandwidth Efficiency in Optical Burst Switching and Optical Circuit Switching Networks", Photonic Network Communications, pp. 297309, 2005.

[2] Hyoung-Il Lee e Seung-Woo Seo, "Hybrid Optical Transport Network (HOTNET): An Optical Network with Hybrid Switching Technologies for Integrated Services", IEICE Trans. Commun., Vol. E88-B, No.10, pp.3874-3885, outubro de 2005.

[3] E. W. M. Wong e M. Zukerman, "Analysis of an Optical Hybrid Switch”, IEEE Communications Letters, Vol. 10, pp. 108-110, No. 2, fevereiro de 2006.

[4] F. Xue, S. J. B. Yoo, H. Yokoyama e Y. Horiuchi, "Performance comparison of optical burst and circuit switched networks",Optical Fiber Communication Conference, março de 2005.

[5] M. J. O’Mahony, C. Politi, D. Klonidis, R. Nejabati e D. Simeonidou, "Future Optical Networks", Journal of Lightwave Technology, pp. 4684-4696, Vol. 24, No. 12, dezembro de 2006.

[6] A. Zalesky, H. L. Vu, E. W. M. Wong, M. Zukerman, Z. Rosberg e M. S. Bilgrami, "Performance evaluation of a hybrid optical switch" Proc. ITC19, pp. 1385-1394, agosto de 2005.

[7] Helvécio M. A. Neto, Eduardo M. G. de Queiroz, Amílcar C. César, "Análise de Rede Brasileira de Comunicação Óptica Integrada com Rede de Satélites LEO", SBrT'08, pp. 1-6 (CD-ROM), Rio de Janeiro, RJ, 2 a 5 de setembro de 2008. 